\title{
Captura de peces no comerciales con espinel experimental de fondo en Isla Gorgona, Colombia, y su área de influencia
}

\author{
Stella Gómez ${ }^{1}$, Julián Alejandro Caicedo ${ }^{1} \&$ Luis Alonso Zapata ${ }^{1 *}$ \\ 1.Programa Marino Costero,WWF Colombia, Cali, Colombia; stellitagomezg@hotmail.com, julian_1@hotmail.com, \\ lazapata@wwf.org.co
}

Recibido 18-X-2013. Corregido 20-XI-2013. Aceptado 19-XII-2013.

\begin{abstract}
Non-commercial fish catches with experimental bottom long line fishing in Gorgona Island, Colombia, and its surroundings areas. Eels and moray eels are among the species that lack commercial value but that are important as bait. However, little information is available about Colombian fishing by-products. Between 2005 and 2009, field work was done in Gorgona National Natural Park and its surrounding areas, with a fishing line made of traditional "J" and circular or curve hooks. We analyzed the catch composition, comparing both types of hooks, emphasizing whether the circular hook modified or not non-commercial captures. Overall, 189 fishing throws where analyzed, standardizing the Catch Per Effort (CPUE) in number of individuals and in kilograms per 100 hooks per hour of task. A total of 50 species where captured, of which 14 are part of the noncommercial category. There were no significant differences for hook types ( $\mathrm{U}=25228, \mathrm{P}>0.1)$, and Gymnothorax equatorialis and Echiophis brunneus were the most abundant species. Both were concentrated in the northern sector of the study area, a rocky banks sector. The mean sexual maturity size was $57.0 \mathrm{~cm}$ in G. equatorialis, and at 85.6cm in E. brunneus. Rev. Biol. Trop. 62 (Suppl. 1): 391-405. Epub 2014 February 01.
\end{abstract}

Key words: bottom line fishing, hooks, protected areas, anguiliformes, Gymnothorax equatorialis, Echiophis brunneus, Colombian Pacific Ocean.

En la zona de influencia del Parque Nacional Natural Gorgona se usan diferentes artes de pesca, siendo el espinel de fondo el más usado. Este arte conocido también como palangre o línea de fondo tiene como objetivo de pesca los recursos demersales, los cuales son organismos asociados al fondo marino somero o profundo para los cuales este constituye su hábitat permanente o temporal ya sea con fines de reproducción y/o alimentación. Están integrados por una gran diversidad de especies, al tiempo que son objeto de aprovechamiento y sustento de distintas pesquerías en el Pacífico colombiano (Rojas \& Zapata, 2006). Dentro de las especies objetivo de esta pesca se encuentran principalmente merluza, cherna, pargos, pero a pesar de ser un arte con cierta selectividad (especies y tallas), se registran capturas incidentales como es el caso de las tortugas marinas.
Desde 2003 en Latinoamérica, WWF se sumó a la Fundación Packard y la Comisión Interamericana del Atún Tropical-CIAT, en una campaña regional (países del Océano Pacífico Oriental-OPO) para introducir nuevas técnicas y cambios en las prácticas de pesca, como parte de una estrategia integral que contribuyera a reducir la mortalidad de tortugas marinas por captura incidental en actividades pesqueras (Zapata et al., 2010). En Colombia el programa inició en el año 2005 introduciendo el anzuelo circular por medio de experimentos donde se intercalan estos con el tradicional, bajo el marco del programa "Disminución de captura incidental de tortugas marinas por medio del anzuelo curvo" (programa BYCATCH) el cual se viene desarrollando en diferentes áreas del Pacífico colombiano, convirtiéndolo en una estrategia viable para el desarrollo de una 
buena práctica pesquera, ya que la captura obtenida con anzuelo circular es comparable con la obtenida con anzuelos tradicionales, es decir no la reduce significativamente; de igual manera disminuye la captura incidental de tortugas y las capturadas tienen una alta tasa de sobrevivencia porque los enganches son menos letales que con el anzuelo J (Zapata et al., 2010).

Aunque los estudios sobre esfuerzos pesqueros se concentran en las especies objeto de interés comercial, la captura generalmente incluye especies que no están en este grupo y son catalogadas como no comerciales o de descarte (Williams, 1974). Dentro del grupo de las especies de descarte que son capturadas con el espinel, se destacan las anguilas y morenas. Estas especies son importantes para la actividad ya que se utilizan como carnada por parte de los pescadores. Sin embargo, no se dispone de mucha información de este grupo.

En la literatura científica los trabajos publicados sobre el orden Anguilliformes son escasos, destacándose el trabajo de Fishelson (1992), quien realizó un estudio comparativo de la morfología gonadal y la sexualidad de la familia Muraenidae en Hawai, y la descripción y registro sistémico de las diferentes especies en el Pacífico Oriental Tropical realizados por Allen \& Robertson (1994), McCosker \& Resonblatt (1995), Aguilar-Palomino, Mariscal-Romero, González-Sansón \& RodríguezIbarra (1996) y Ruiz-Ramírez et al. (1997), incluyendo la comparación con nuevas especies (Lavenberg, 1992; Hatooka \& Randall, 1992; Bussing, 2000).

Para el PNN Gorgona la información existente se limita exclusivamente a aspectos taxonómicos y sistemáticos, a excepción de los trabajos de grado realizados por Rivas (1998), quien hizo un estudio preliminar sobre la ecología trófica de algunas especies de peces capturadas en el PNN Gorgona y Sanquianga, dentro de las cuales está incluida Gymnothorax equatorialis; Zorrilla (1999), quien realizó un estudio de los aspectos reproductivos de Gymnothorax sp. aff equatorialis y Ophichthus pacifici, y el de García (2005), quien analizó los aspectos biológicos y pesqueros de tres especies de anguiliformes: Gymnothorax equatorialis, Ophichthus frontalis y Echiophis brunneus.

El presente estudio brinda información de la composición de las especies ícticas no comerciales capturadas con espinel tradicional, haciendo énfasis en tallas y reproducción de las más abundantes de esta categoría, $G$. equatorialis y E. brunneus, evaluando además si existen diferencias en captura entre el anzuelo tradicional " $\mathrm{J}$ " y el circular "C12/0", con el propósito de contribuir al conocimiento de estas especies, las cuales hacen parte de los recursos demersales presentes en el área marina protegida del PNN Gorgona. Además se genera información de importancia para un área estratégica del sector sur del Pacífico colombiano, en la cual se encuentran dos parques nacionales naturales, que generan un ordenamiento especial para este sector.

\section{MATERIALES Y MÉTODOS}

Área de estudio: Los monitoreos se realizaron a manera de pesca experimental en El PNN Gorgona, ubicado en el Océano Pacífico Oriental $\left(02^{\circ} 49^{\prime}-03^{\circ} 0^{\prime} \mathrm{N}\right.$ y $\left.78^{\circ} 06^{\prime}-78^{\circ} 18^{\prime} \mathrm{W}\right)$, a $27 \mathrm{~km}$ del continente, y al frente de Punta Reyes donde se ubica la localidad de Bazán, la cual hace parte del PNN Sanquianga. Dentro del área protegida del PNN Gorgona se encuentran los denominados bancos de pesca del norte y del sur de la isla, importantes sitios de captura de diferentes recursos (UAESPNN, 1998).

Los monitoreos fueron realizados de manera mensual durante tres días entre el 2005 y 2009 dentro del PNN Gorgona y en su área de influencia. Para los monitoreos se usó un espinel experimental donde se alternaron en igual número anzuelos tradicionales "J" número 7/8 (J7) y anzuelos circulares número 12/0 (C12) en una proporción 1:1 (Fig. 1). Las faenas de captura se realizaron principalmente en la noche ya que el espinel experimental se caló generalmente en horas de la tarde y se levantó en las primeras horas de la mañana del siguiente día.

Procesamiento de capturas: El producto obtenido durante los muestreos fue identificado 
A

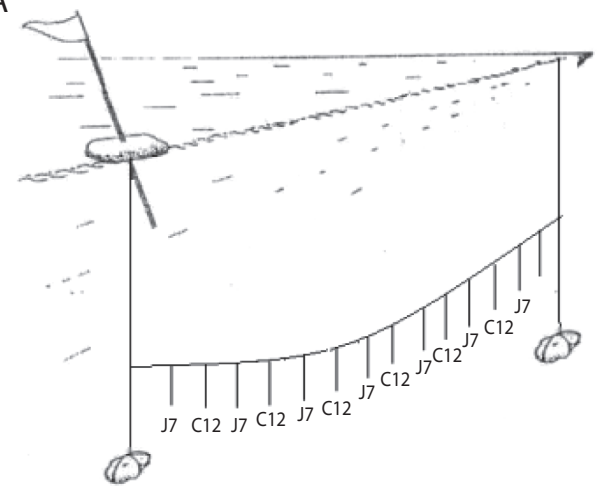

B

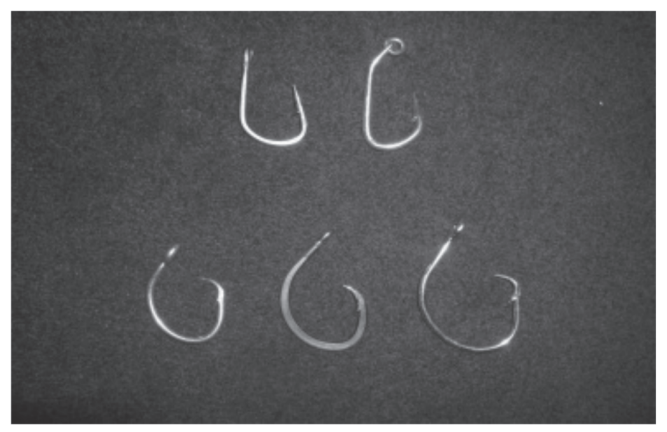

Fig. 1. A. Espinel experimental de fondo utilizado en los monitoreos; B. Anzuelo tradicional (arriba) y anzuelo curvo (abajo). Fig. 1. A. Experimental bottom long-line used in the monitoring: B. traditional hook (above) and circle hook (below).

siguiendo las claves para peces del Pacífico Oriental (Allen \& Robertson, 1994; Fischer, Krupp \& Schneider, 1995). Se registraron datos merísticos y biológicos, tales como longitud total (Lt), longitud estándar (Ls), peso total (Wt), peso de vísceras, gónadas (Wg), hígado (Wh), sexo y estado de madurez cuando fue posible. En los años 2005-2007 el procesamiento de la captura obtenida durante los monitoreos se realizó en tierra, en el PNN Gorgona, la mayoría de las veces de manera directa por un biólogo que se embarcaba para tomar la información directamente. En el 2008 y 2009 los datos fueron tomados por un observador entrenado.

Con las capturas obtenidas en el PNN Gorgona y en el área de influencia con el arte de pesca espinel experimental, se determinaron las diferentes especies capturadas con cada anzuelo. Las capturas del espinel experimental se agruparon en tres categorías comerciales, denominadas $\mathrm{Cc} 1$, que corresponde a las especies que tienen un mejor precio en el cuarto frío y pueden hacer parte de cadenas de mercados nacionales e internacionales, Cc2 que corresponde a las especies cuya importancia es local debido a que se comercializan en la comunidad y son utilizadas principalmente como materia prima para procesos de salazón y consumo familiar y $\mathrm{Cc} 3$, que corresponde a las especies cuya finalidad es proveer una alternativa de carnada para la pesca y las que no son devueltas muertas al mar.

Toda la captura se estandarizó con base en el esfuerzo, considerando número de individuos y kilogramo por centena de anzuelos por hora. Se evaluaron las diferencias en la CPUE de las especies de la categoría Cc3 considerando los dos tipos de anzuelos dispuestos en el espinel experimental, mediante una prueba no paramétrica de Mann-Whitney. Se describió la variación espacial de la CPUE en el área de estudio de las dos especies más representativas en la categoría Cc3 (G. equatorialis y $E$. brunneus) usando ArcMap 10, y se realizó una comparación de la distribución de frecuencia de tallas entre estas dos especies.

Se evaluó la diferencia en la CPUE y la madurez sexual de G. equatorialis y E. brunneus considerando el tipo de anzuelo. La talla media de madurez se definió como la talla promedio a la que el $50 \%$ de los individuos están sexualmente maduros, y se estimó utilizando el método descrito por Vazzoler (1981), el cual consiste en ajustar una curva sigmoide a la proporción de individuos sexualmente maduros por intervalos de talla. Adicionalmente, se realizó el cálculo de la talla promedio de primera madurez sexual (M) utilizando el método estadístico propuesto por Udupa (1986), como

$$
M=\operatorname{antilog}\left[X_{k}+\frac{X}{2}-\left(X \sum p_{i}\right)\right]
$$


donde Xk: logaritmo del punto medio de la clase de talla donde todos los individuos están completamente maduros, X: logaritmo del incremento promedio, Pi: Proporción de peces completamente maduros en el i-ésimo grupo. Se emplearon los límites de confianza del $95 \%$ como:

$$
\operatorname{antilog}\left[\mathrm{m} \pm 1.96 \sqrt{X^{2} \sum\left(\frac{p_{i} x q_{i}}{n_{i}-1}\right)}\right]
$$

donde $\mathrm{m}$ : logaritmo de $\mathrm{M}$, pi : número de maduros en el iésimo grupo/tamaño muestreado para cada grupo, qi : 1-pi, y ni : tamaño muestreado para cada grupo.

\section{RESULTADOS}

Entre los años 2005 y 2009 se realizaron 189 lances con espinel experimental para un total de anzuelos evaluados de 38960 anzuelos C12 y 38960 para J7. Del total de lances, 73 fueron calados en el PNN Gorgona y 116 en su área de influencia. Se capturaron 2155 individuos de las tres categorías ( 1104 con $\mathrm{C} 12$ y 1 051 con J7), para un total de $4029 \mathrm{~kg}(2213 \mathrm{~kg}$ con $\mathrm{C} 12$ y $1816 \mathrm{~kg}$ con J7).

De las 50 especies registradas (46 con $\mathrm{C} 12$ y 40 con J7), 36 fueron capturadas por los dos tipos de anzuelos, las 14 restantes son consideradas como especies esporádicas que fueron capturadas por uno u otro anzuelo. A pesar que el anzuelo curvo presentó más especies capturadas (diez exclusivamente capturadas con anzuelo $\mathrm{C}$, contra cuatro capturadas exclusivamente con anzuelo J), estas especies son poco frecuentes con capturas entre uno y cinco individuos (Fig. 2).

En la composición por anzuelo se observó que en el anzuelo tradicional la especie que ocupó el primer lugar fue G. equatorialis con un $19,6 \%$, seguido por B. clarkae con $10,9 \%$ (Fig. 3A) y con el anzuelo curvo esta especie baja a un segundo lugar $(12,86 \%)$ y $B$. clarkae pasa a ocupar el primer lugar (17,75\%) (Fig. 3B).

En las Fig. 4 y 5 se presenta la CPUE en número de individuos y peso, respectivamente, por año de estudio para los dos anzuelos. Para la categoría tres el aporte en número es mayor para los dos anzuelos comparados con el peso y se observa una tendencia de captura superior con el anzuelo tradicional. La categoría tres no mostró diferencias significativas entre anzuelos para la CPUE ni en número ( $\mathrm{U}=25228$, $\mathrm{p}>0.1)$ ni en peso $(\mathrm{U}=25878, \mathrm{p}>0.1)$ (Fig. $6 \mathrm{~A}$ y B). Esta categoría estuvo compuesta por 14 especies de las cuales ocho pertenecen al orden

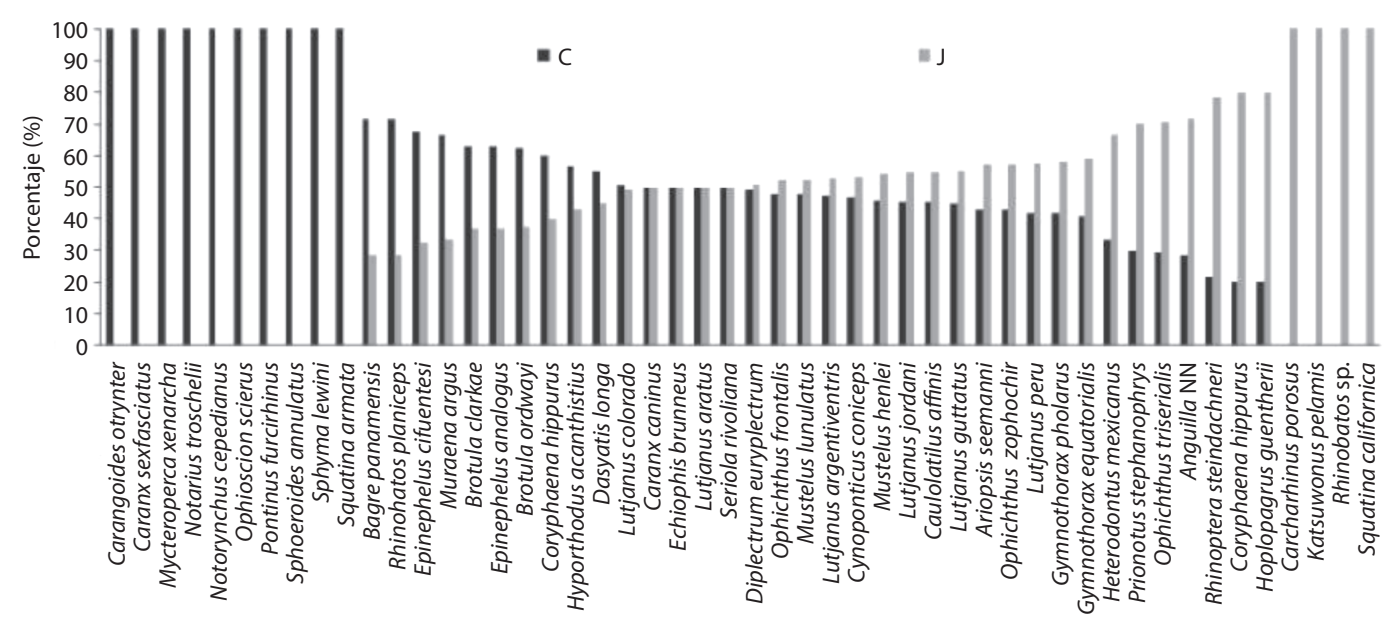

Fig. 2. Porcentaje en número de las especies capturadas con el espinel experimental, de acuerdo al tipo de anzuelo. Fig. 2. Percentage number of species caught with the experimental bottom long-line, according to the type of hook. 

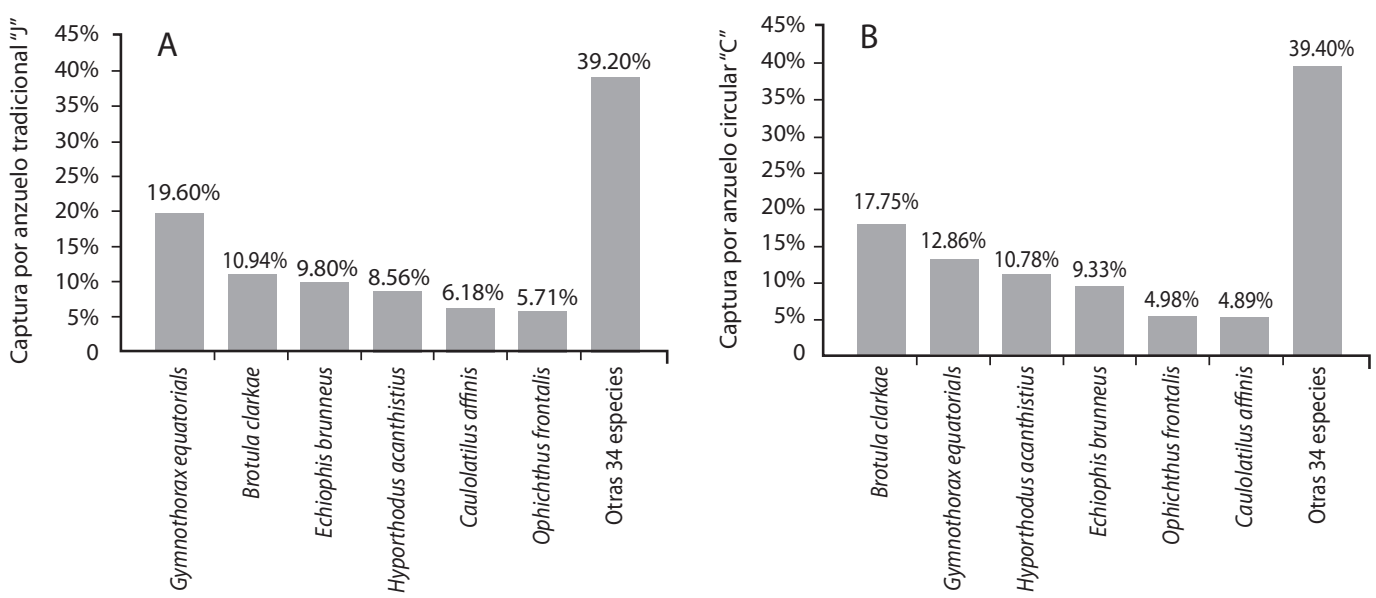

Fig. 3. Composición de captura por anzuelo. A: Anzuelo tradicional "J"; B: Anzuelo circular "C".

Fig. 3. Composition of catch per hook. A: traditional hook "J": Circle hook "C".

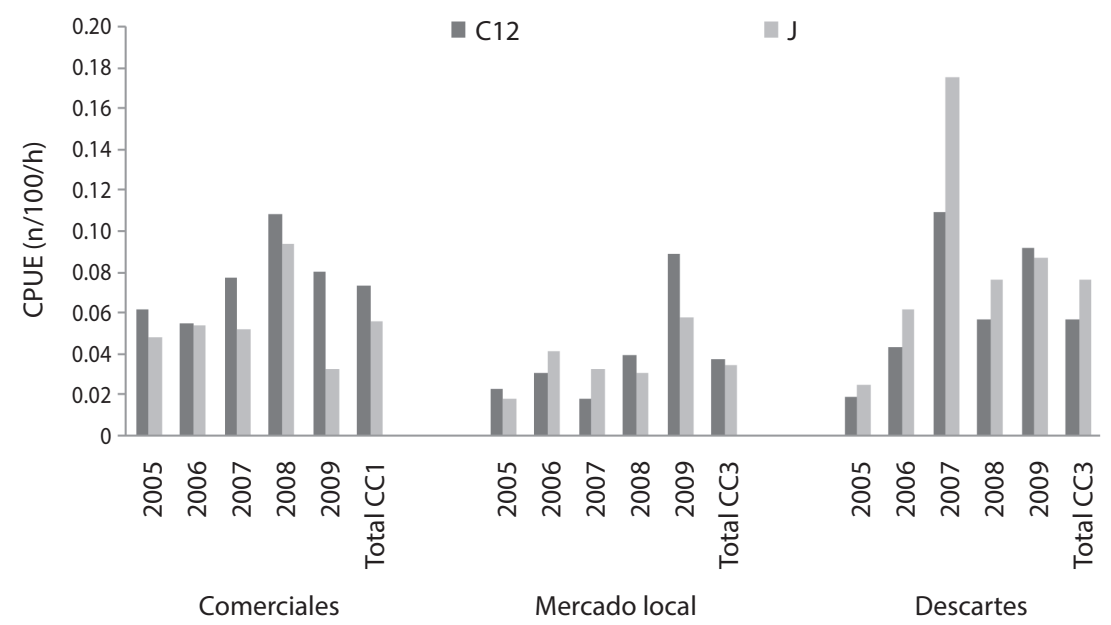

Fig. 4. Variación anual de la CPUE en número de individuos para las tres categorías.

Fig. 4. Annual variation in number of individuals (CPUE) for the three categories.

anguiliformes, las otras seis fueron especies capturadas de forma esporádica con menos de cinco individuos, siendo las especies más representativas Gymnothorax equatorialis con 306 individuos $(\mathrm{C}=120$ y $\mathrm{J}=186)$ y Echiophis brunneus con $144(\mathrm{C}=77 \mathrm{y} \mathrm{J}=67)$ (Cuadro 1).

Al realizar el análisis espacial de la CPUE para G. equatorialis se observaron concentraciones medias (entre 0.042 y 0.067 ) y altas $(0.067$ y 0.126$)$ hacia la zona norte de isla Gorgona. Para E. brunneus no se observó una zonificación definida, y los valores de las concentraciones se encontraron en su mayoría en categoría media $(0.027$ y 0.056$)$ y alta $(0.056$ y 0.0) (Figs. 7 y 8 ).

No se encontraron diferencias significativas entre los anzuelos para la CPUE en peso y en número de $G$. equatorialis ( $\mathrm{U}=10$ 888, $\mathrm{p}>0.1),(\mathrm{U}=13$ 207, $\mathrm{p}>0.1)$ y de E. brunneus $(\mathrm{U}=2$ 076, $\mathrm{p}>0.1),(\mathrm{U}=4$ 877, $\mathrm{p}>0.1)$ respectivamente (Fig. 9A, B, C y D). La talla media de madurez se obtuvo para toda la población 


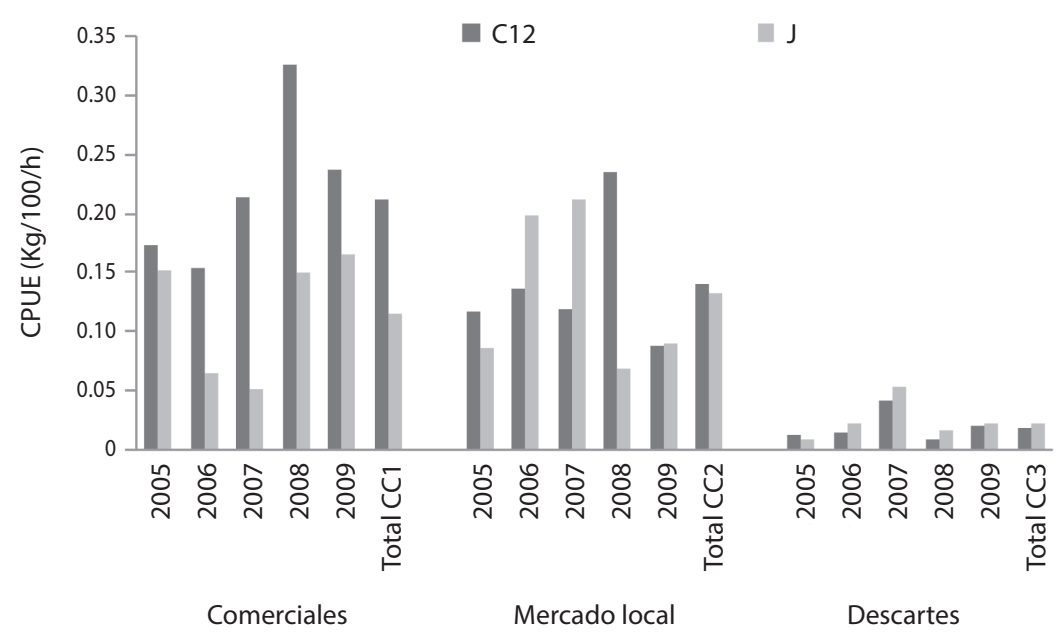

Fig. 5. Variación anual de la CPUE en peso para las tres categorías.

Fig. 5. Annual variation in weight (CPUE) for the three categories.
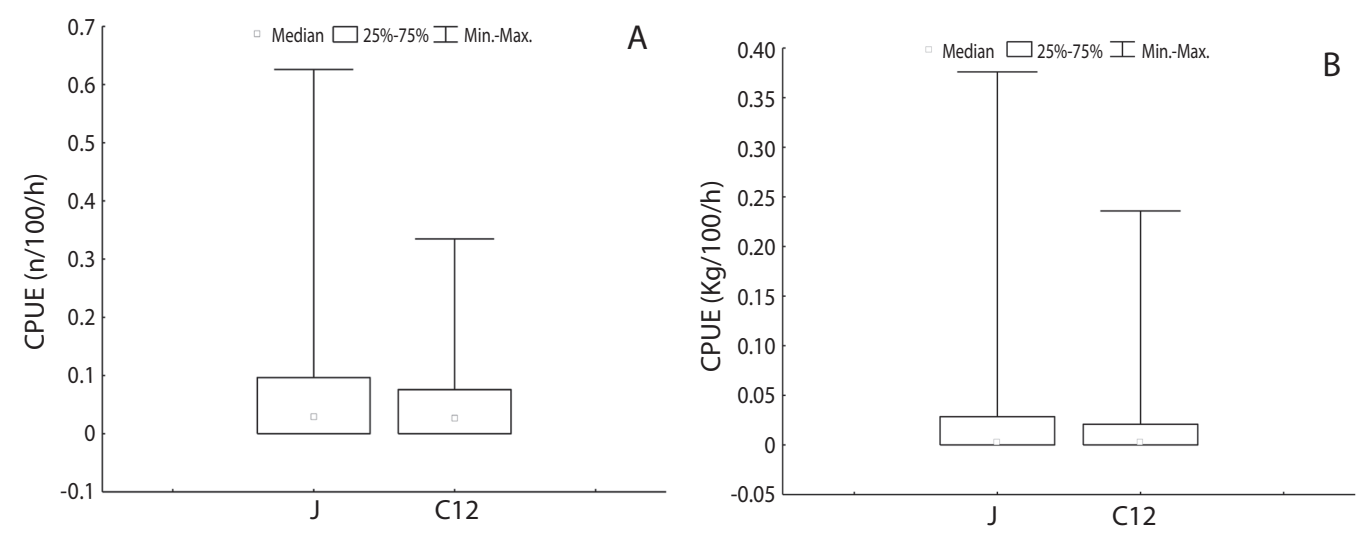

Fig. 6. Análisis de CPUE (mediana \pm Min-Máx) entre anzuelos $J$ y curvo de la categoría comercial tres: (A) CPUE en número; (B) CPUE en peso.

Fig. 6. Analysis of CPUE (median \pm Min-Max) between traditional hook and circle hook of the commercial category three: (A) CPUE in number; (B) CPUE in weight.

y no se discriminó por anzuelo por ser un dato que caracteriza la población. La talla media de madurez sexual estimada por el método grafico fue de $57.0 \mathrm{~cm} G$. equatorialis y de $85.6 \mathrm{~cm} E$. brunneus (Fig. 10A y B). Los valores obtenidos por Udupa (1986) para la talla de primera madurez sexual en $G$. equatorialis fue de $53.8 \mathrm{~cm}$ (IC95\%: 44.0-65.6cm) y para E. brunneus fue de $69.3 \mathrm{~cm}$ (IC95\%: 58.4-82.2) (Fig. 11 A y B). Tampoco se detectaron diferencias significativas para la talla media de captura de estas dos especies entre los dos tipos de anzuelos utilizados en el espinel experimental $G$. equatorialis $(\mathrm{U}=10710, \mathrm{p}>0.1)$ y E. brunneus $(\mathrm{U}=2$ 077, $\mathrm{p}>0.1)$.

Al hacer el análisis de la distribución de frecuencia de tallas del anzuelo curvo y el tradicional para las dos especies se encontró que el $66.7 \%$ de los individuos de $G$. equatorialis que fueron capturados con anzuelo circular estuvieron por encima de la primera talla de madurez; mientras que el $85.5 \%$ de 
CUADRO 1

Talla mínima, máxima y promedio $(\mathrm{cm})$ para la Cc3 por anzuelo

TABLE 1

Minimum size, maximum and average $(\mathrm{cm})$, for Cc 3 by type of hook

\begin{tabular}{|c|c|c|c|c|c|c|c|c|}
\hline \multirow{3}{*}{ Especie } & \multicolumn{8}{|c|}{ Anzuelo } \\
\hline & \multicolumn{4}{|c|}{$\mathrm{C} 12$} & \multicolumn{4}{|c|}{$\mathrm{J}$} \\
\hline & Mín & Max & Prom & $\mathrm{n}$ & Mín & Max & Prom & $\mathrm{n}$ \\
\hline Anguilla NN & 62,5 & 66,5 & 64,5 & 2 & 45 & 79,5 & 63,7 & 5 \\
\hline Echiophis brunneus & 58,9 & 119,5 & 88,2 & 77 & 43 & 130 & 87,2 & 67 \\
\hline Gymnothorax equatorialis & 37,5 & 100 & 57,3 & 120 & 37,9 & 94,5 & 56,1 & 186 \\
\hline Gymnothorax phalarus & 40 & 81,5 & 58,7 & 11 & 45 & 85,2 & 61,0 & 16 \\
\hline Heterodontus mexicanus & & & & 1 & & & & 2 \\
\hline Muraena argus & 71 & 87,5 & 79,3 & 2 & 68,5 & 74 & 71,8 & 3 \\
\hline Ophichthus frontalis & 43,2 & 88,7 & 61,5 & 53 & 39,2 & 93,5 & 61,3 & 55 \\
\hline Ophichthus triseria/is & 38 & 83 & 62,9 & 5 & 73 & 120 & 88,4 & 12 \\
\hline Ophichthus zophochir & 47,5 & 79,3 & 62,0 & 17 & 50,2 & 76 & 59,0 & 24 \\
\hline Pontinus furcirhinus & 21,5 & 21,5 & 21,5 & 1 & & & & \\
\hline Prionotus stephanophrys & 35 & 36,7 & 35,9 & 2 & 30 & 37 & 33,9 & 7 \\
\hline Sphoeroides annulatus & 36,5 & 36,5 & 36,5 & 1 & & & & \\
\hline Squatina armata & 95,5 & 95,5 & 95,5 & 1 & & & & \\
\hline Squatina californica & & & & & 96 & 96 & 96,0 & 1 \\
\hline
\end{tabular}

los capturados con el anzuelo J estuvieron por encima de la primera talla de madurez sexual. En E. brunneus el $87 \%$ de los individuos capturados con anzuelo curvo estuvieron por encima de la primera talla de madurez; mientras que el $85.4 \%$ de los individuos capturados con anzuelo J estuvieron por encima de la talla de madurez sexual. Este resultado se hace más evidente con el análisis espacial para cada especie, considerando los individuos maduros e inmaduros y comparando el anzuelo J contra el curvo (Fig. 12 y 13). En este sentido, se están capturaron más individuos maduros de G. equatorialis con el anzuelo $\mathrm{J}$ que con el circular, mientras que para E. brunneus no se detectó una diferencia significativa entre anzuelos, aunque fue ligeramente mayor la captura de individuos maduros con el anzuelo circular.

\section{DISCUSIÓN}

La composición de especies capturadas con el espinel experimental no presentó diferencias significativas asociadas al tipo de anzuelo utilizado. La mayoría de especies fueron capturadas por los dos anzuelos y las que no, son especies con baja frecuencia de ocurrencia. Los resultados de la CPUE, tanto en número como en peso sugirieron que las capturas con el anzuelo curvo fueron menores que con el anzuelo $\mathrm{J}$ en la categoría tres (contrario a las categorías uno y dos), lo cual sería un beneficio ecológico para la fauna íctica que en su mayoría no tiene un valor comercial, sin embargo estas diferencias no fueron estadísticamente significativas. Por lo tanto, este es un tema que debe continuar bajo análisis experimental, pues el presente trabajo no permite comprobar la diferencia entre los dos anzuelos, diferencia que es expresada continuamente por los pescadores.

En las especies más representativas de la categoría $\mathrm{Cc} 3$ es evidente la disminución para $E$. brunneus y $G$. equatorialis en la composición de la captura con el anzuelo circular, aunque esta diferencia tampoco fue estadísticamente significativa. En la geo-referenciación de la CPUE para las dos especies se identificó para $G$. equatorialis una posible preferencia por la zona norte del PNN Gorgona, conocida como los bancos rocosos del norte que se 


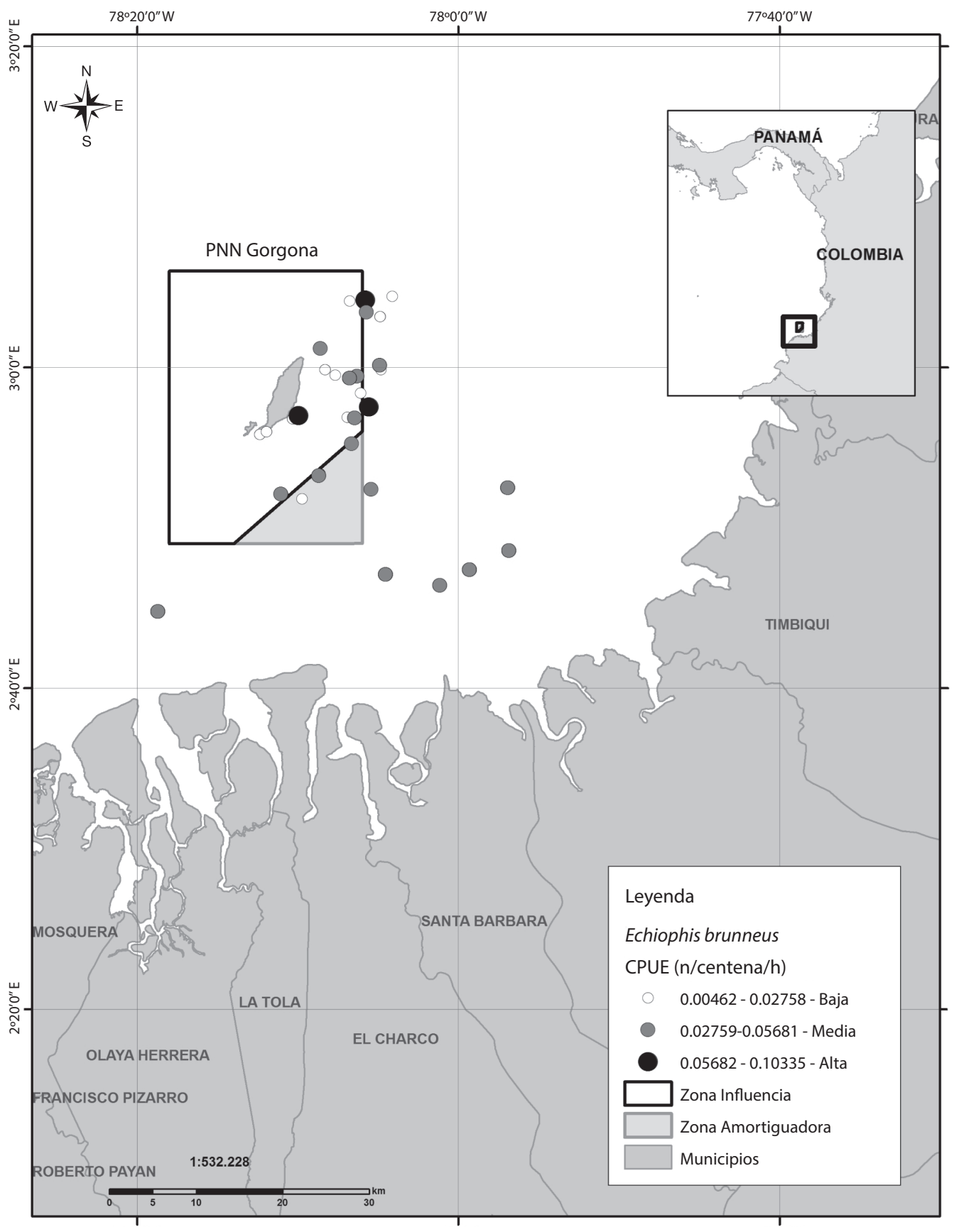

Fig. 7. Análisis geográfico de la CPUE en número para la especie G. equatorialis.

Fig. 7. Geographic analysis of CPUE in number for the specie G. equatorialis. 


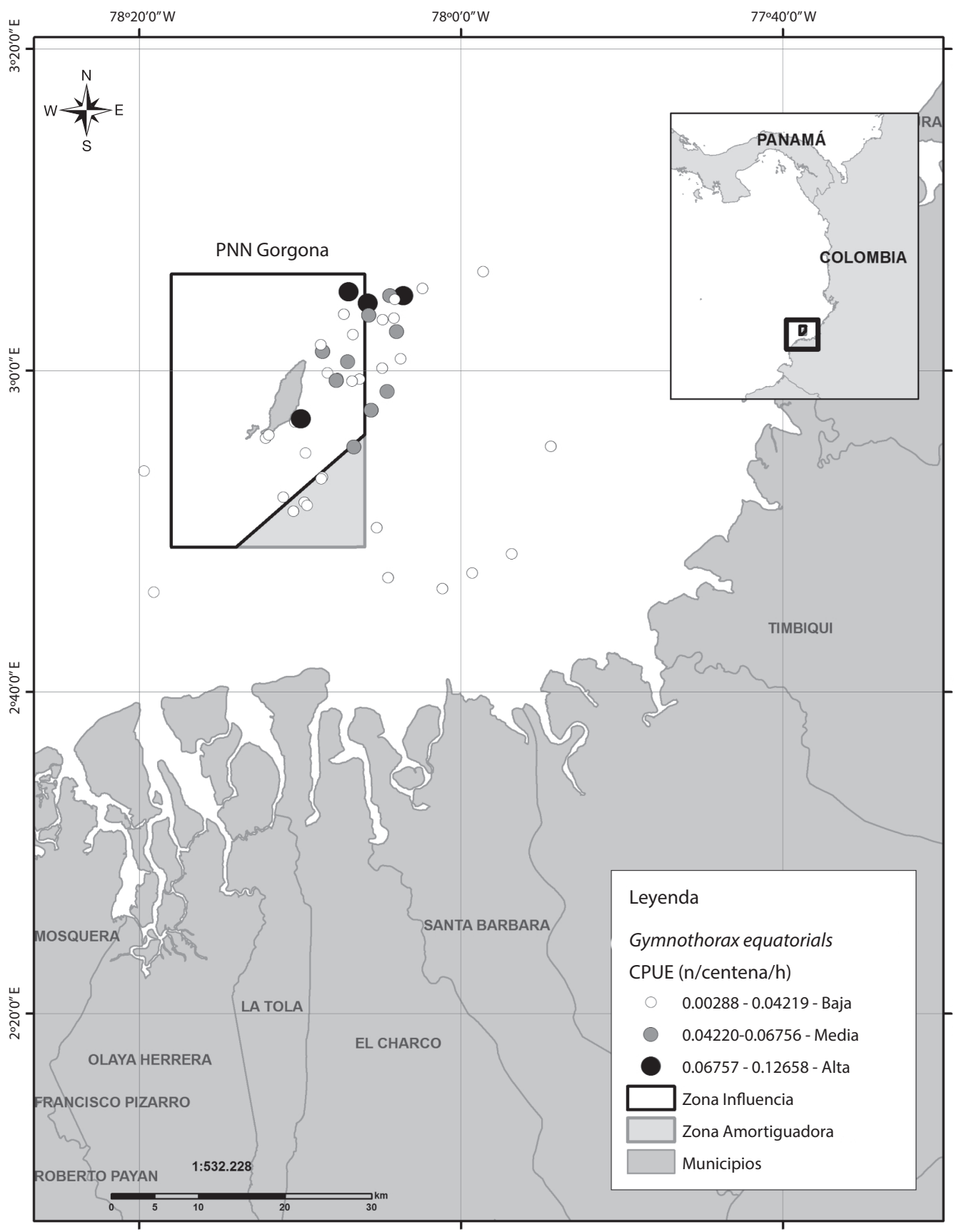

Fig. 8. Análisis geográfico de la CPUE en número de la especie E. brunneus.

Fig. 8. Geographic analysis of CPUE in number for the specie E. brunneus. 

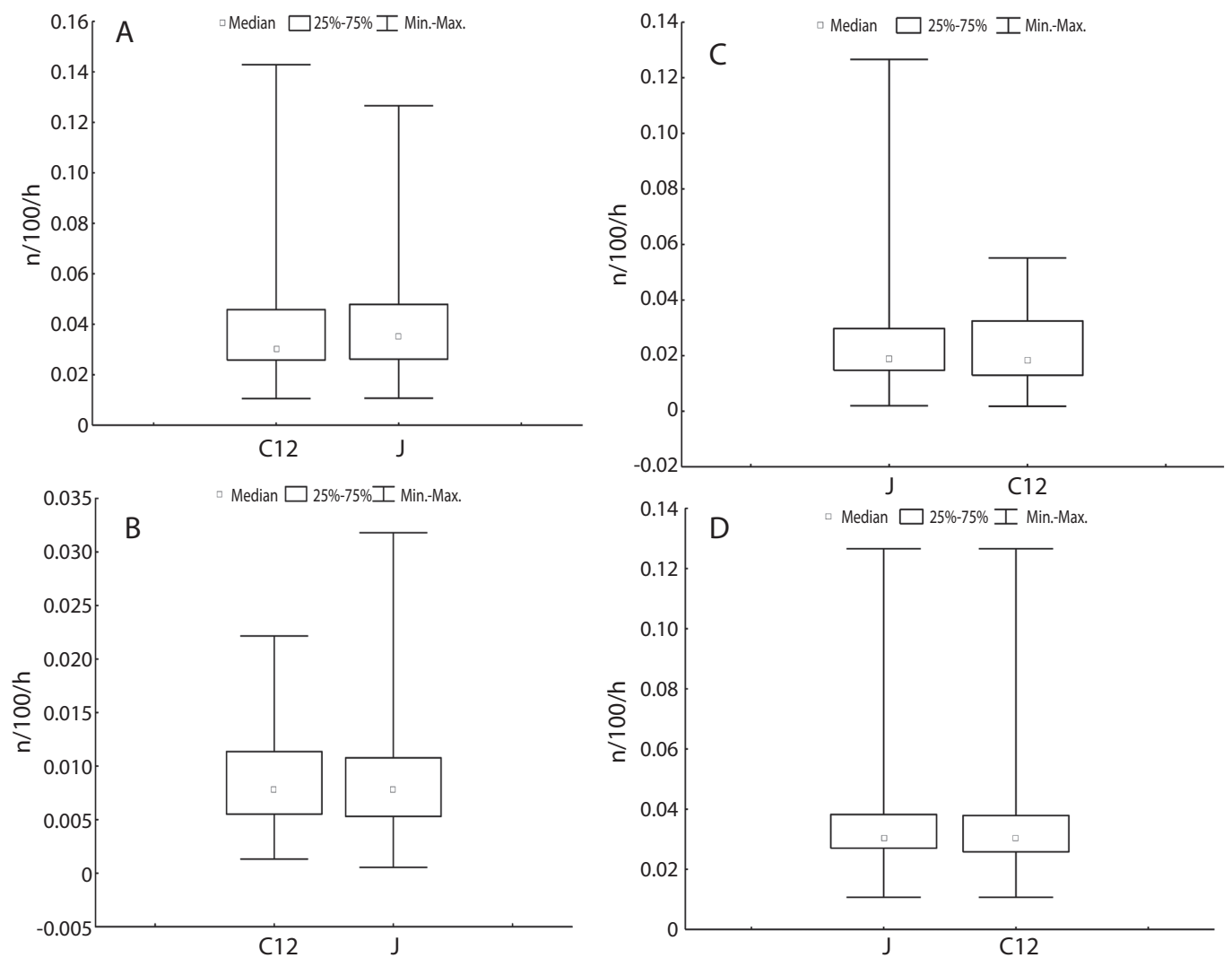

Fig. 9. Análisis de CPUE (mediana \pm Min-Máx) entre anzuelos J y curvo: (A) CPUE peso G. equatorialis; (B) CPUE en número G. equatorialis; (C) CPUE en peso E. brunneus; (D) CPUE número E. brunneus.

Fig. 9. Analysis of CPUE (median \pm Min-Max) between traditional hook and circle hook: (A) CPUE weight G. equatorialis; (B) CPUE in number G. equatorialis; (C) CPUE in weight E. brunneus; (D) CPUE in number E. brunneus.
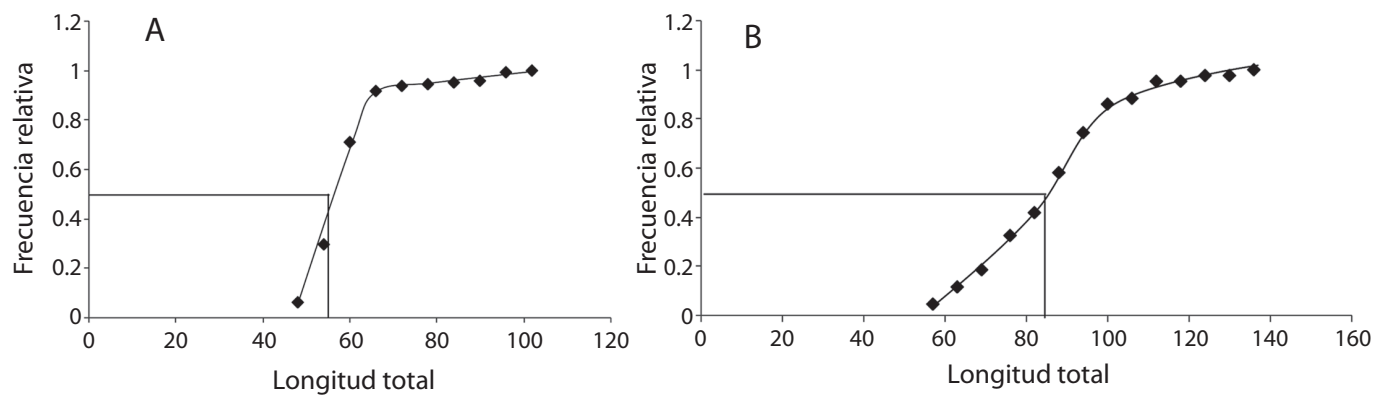

Fig. 10. Talla media de madurez; A: G. equatorialis, B: E. brunneus.

Fig. 10. Size at average maturity; A: G. equatorialis, B: E. brunneus. 

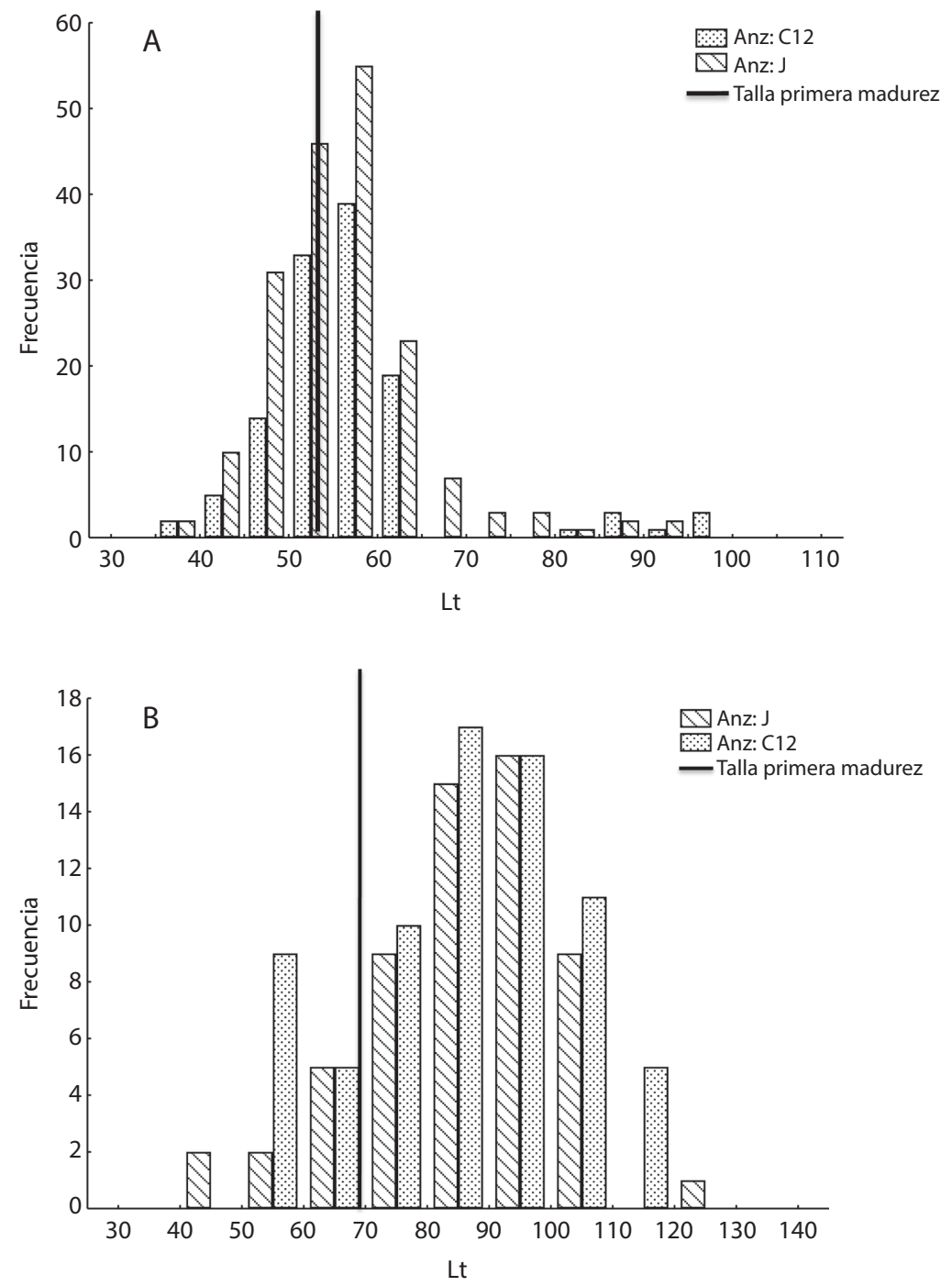

Fig. 11. Distribución de frecuencias para los dos anzuelos con la talla media de madurez A: G. equatorialis; B: E. brunneus. Fig. 11. Frecuency distribution for the two hooks with size at average maturity, A: G. equatorialis; B: E. brunneus.

caracteriza por ser una zona importante de pesca en el Pacífico colombiano (Zapata et al., 1999) y que valdría la pena muestrear con mayor intensidad para saber si corresponde a un área de importancia biológica para la especie (refugio, alimentación, reproducción o algún otro aspecto de historia de vida). Esta zona está dentro de los objetivos de ordenamiento del PNN Gorgona debido a que parte de este banco está en la zona interior del área protegida (UAESPNN, 1998). Con respecto a la identificación de áreas de reproducción para estas dos especies con los datos geo-referenciados el análisis no mostró una zona definida.

La talla media de madurez sexual establecida para G. equatorialis en este estudio fue concordante con lo reportado por García (2005) en el PNN Gorgona y su área de influencia. Sin embargo, Lucano-Ramírez, Ruiz-Ramírez, Rojo-Vázquez \& González-Sansón (2008), 

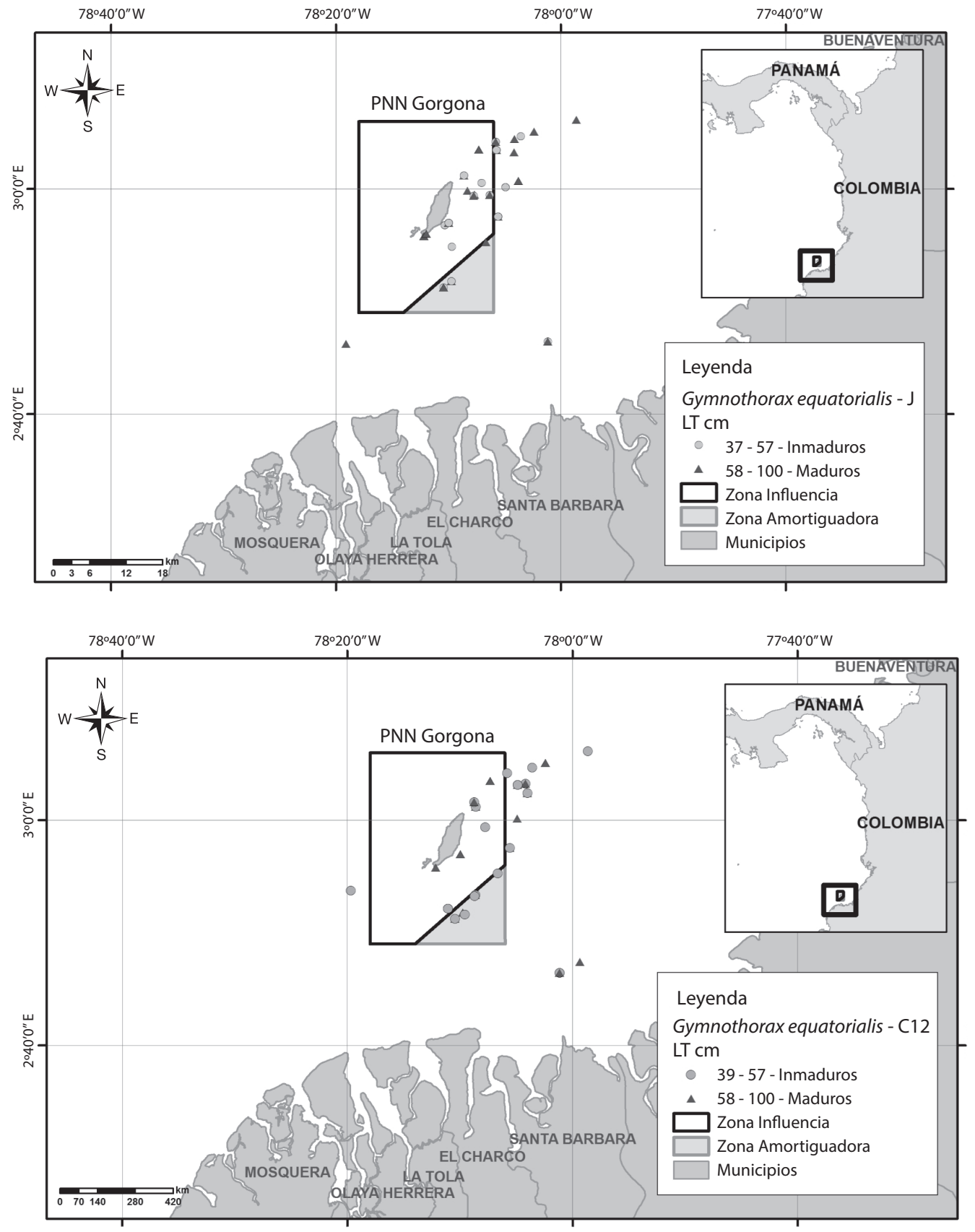

Fig. 12. Análisis geográfico para G. equatorialis de los individuos maduros e inmaduros, por tipo de anzuelo. Fig. 12. Geographic analysis of the mature and immature individuals for G. equatorialis, by type of hook. 

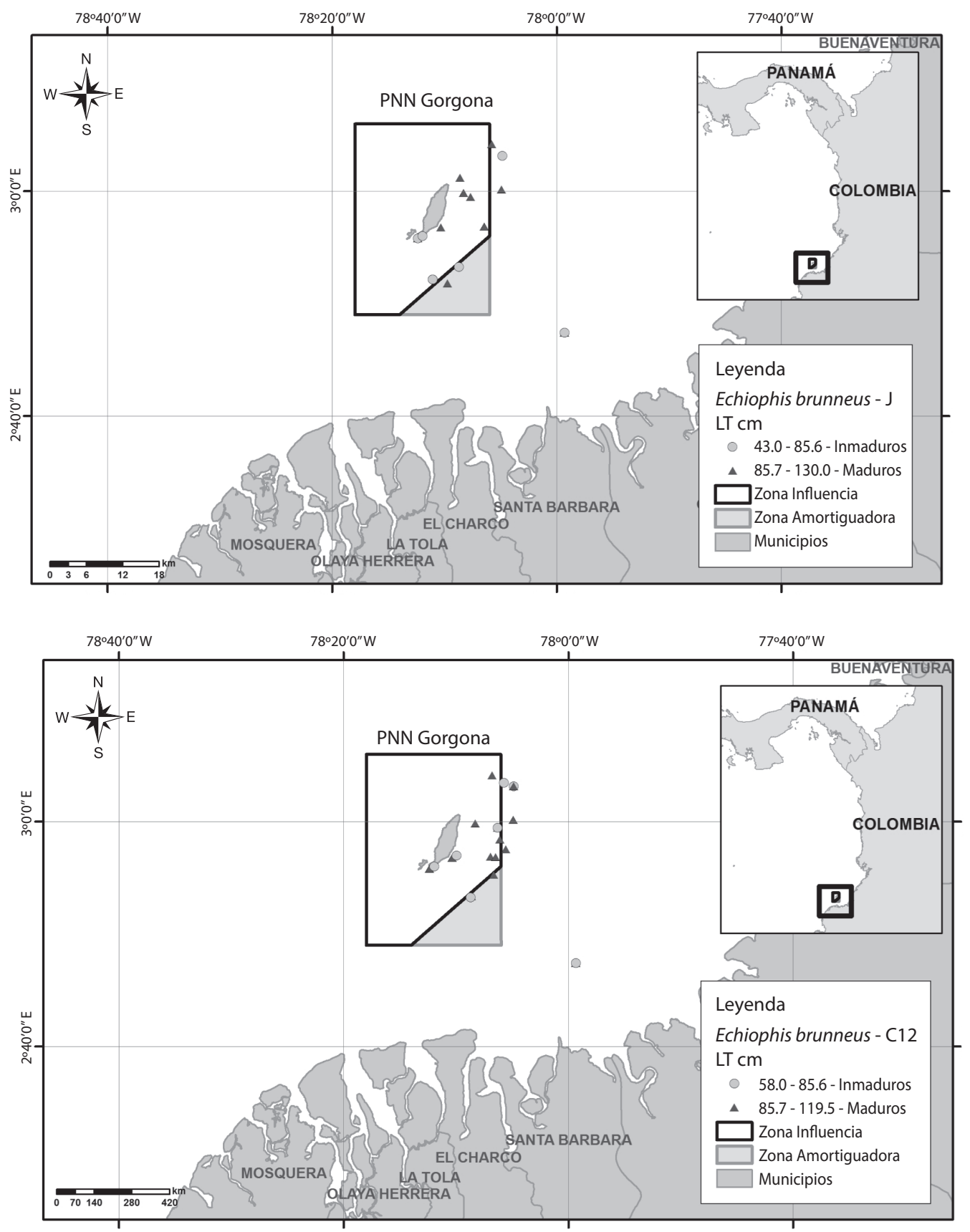

Fig. 13. Análisis geográfico para E. brunneus de los individuos maduros e inmaduros por tipo de anzuelo.

Fig. 13. Geographic analysis of the mature and immature individuals for E. brunneus, by type of hook. 
reportó para México una talla media de madurez para hembras de $43.7 \mathrm{~cm}$ y para machos de $42.7 \mathrm{~cm}$. Probablemente, aspectos biogeográficos pueden estar estructurando las características poblacionales para cada uno de los grupos. Por otra parte, la talla media de madurez sexual establecida para E. brunneus en este estudio fue concordante con lo reportado por García (2005) para esta especie siendo de $87 \mathrm{~cm}$ según este autor.

Es necesario destacar que se recomienda considerar las estimaciones de talla de primera madurez sexual realizadas con el método numérico de Udupa (1986), ya que esta aproximación permite definir un intervalo de confianza para el estimador. Al realizar los análisis estadísticos no se encontraron diferencias significativas entre anzuelos, sin embargo es de resaltar que los dos anzuelos están capturando estas dos especies por encima de la primera talla de madurez sexual. Esto es importante para la sostenibilidad del recurso, sin embargo se debe seguir evaluando el comportamiento de estos valores no solo en las especies trabajadas sino en toda la categoría tres por la posibilidad de que esta pueda llegar a tener un aprovechamiento dirigido, lo anterior debido a que las dinámicas comerciales de los recursos pueden a futuro presionar explotación de la categoría y se crearía así una pesca dirigida hacia algunos de estos recursos como sucede en otros países.

Finalmente las estrategias de ordenamiento que sean desarrolladas a futuro para esta zona, donde en promedio se puede estimar un número de 30000 anzuelos calados diariamente (Castillo, en prep.), deben considerar la evaluación del grupo de categoría comercial tres y el uso de los anzuelos circulares como una nueva alternativa, no solo por la disminución de captura en tortugas marinas sino por la percepción de los pescadores en cuanto a la disminución de captura de las anguilas y morenas. A pesar que en el estudio no se detectó diferencias estadísticas significativas entre los anzuelos, se puede estar generando un valor ecológico en dicha diferencia a favor del circular (menos fauna incidental capturada), por esta razón se recomienda continuar con el esfuerzo de monitoreo en estas localidades.

\section{AGRADECIMIENTOS}

A la comunidad de pescadores de Bazán, el Consejo Comunitario Bajo Tapaje, a los funcionarios del PNN Gorgona y Sanquianga, al programa Bycatch, a Julio Herrera, Paola Mejía, Leidy Cuadros y Verónica Robledo. A los evaluadores externos por sus valiosas contribuciones.

\section{RESUMEN}

Durante los años 2005 a 2009 se realizaron faenas experimentales de pesca en el Parque Nacional Natural Gorgona y su área de influencia, con un espinel compuesto por anzuelos tradicionales $\mathrm{J}$ y circulares o curvos. Este estudio analiza la composición de las capturas comparando los dos anzuelos y enfatiza en si el anzuelo circular modifica o no las capturas no comerciales (descarte). En total se analizaron 189 lances de pesca estandarizándose la captura por unidad de esfuerzo (CPUE) en número de individuos y en kilogramos por 100 anzuelos por hora de faena. Se capturaron en total 50 especies, de las cuales 14 hacen parte de la categoría no comercial o de descarte. No se detectaron diferencias significativas en la CPUE de estas especies considerando el tipo de anzuelo $(\mathrm{U}=25228, \mathrm{p}>0.1)$ siendo Gymnothorax equatorialis y Echiophis brunneus las especies más abundantes Al evaluar el patrón espacial de variación de la CPUE para estas especies, se encontró que ambas se concentran en el sector norte del área de estudio, asociada a la zona de bancos rocosos. Finalmente, se estableció la talla media de madurez sexual para G. equatorialis en $57.0 \mathrm{~cm}$ y de E. brunneus en $85.6 \mathrm{~cm}$.

Palabras Clave: pesca de fondo, anzuelos, áreas protegidas, anguiliformes, Gymnothorax equatorialis, Echiophis brunneus, Pacífico colombiano.

\section{REFERENCIAS}

Aguilar-Palomino, B., Mariscal-Romero, J., GonzálezSansón, G. \& Rodríguez-Ibarra, L. (1996). Ictiofauna demersal de fondos blandos de la plataforma continental de Jalisco y Colima, México, en la primavera de 1995. Ciencias Marinas, 22: 469-481.

Allen, G. R. \& Robertson, D. R. (1994). Fishes of the tropical eastern Pacific. Crawford House Press, Bathhurst, Australia. 
Bussing, W. A. (2000). Gymnothorax phalarus, a new Eastern Pacific moray eel (Pisces: Muraenidae). Revista de Biología Tropical, 46: 439-446.

Fischer, W., Krupp, F., Schneider, W. (1995). (Eds.). Guía FAO para la Identificación de especies para los fines de la Pesca, Pacifico Centro-Oriental (Vol. III. Partes 1 y 2). FAO, Roma, Italia.

Fishelson, L. (1992). Comparative gonad morphology and sexuality of the Muraenidae (Pisces, Teleostei). Copeia, 1: 197-209.

García, A. (2005). Aspectos biológicos y pesqueros de tres especies de anguiliformes: Gymnothorax equatorialis, Ophichthus frontalis y Echiophis brunneus en el Parque Nacional Natural Gorgona y su área de influencia, Pacífico colombiano (Tesis de pregrado) Universidad del Valle, Cali, Colombia.

Hatooka, K. \& Randall, J. E. (1992). A new moray eel (Gymnothorax: Muraenidae) from Japan and Hawaii. Japanese Journal of Ichthyology, 39: 183-190.

Lavenberg, R. J. (1992). A new moray eel (Muraenidae: Gymnothorax) from oceanic islands of the South Pacific. Pacific Science, 46: 58-67.

Lucano-Ramírez, G., Ruiz-Ramírez, S., Rojo-Vázquez, J. A. \& González-Sansón, G. (2008). Reproducción de la morena, Gymnothorax equatorialis (Pisces: Muraenidae) en Jalisco y Colima, México. Revista de Biología Tropical, 56(1): 153-163.

McCosker, J. E. \& Rosenblatt, R. H. (1995). Muraenidae. In W. Fischer, F. Krupp, W. Schneider, C. Sommer, K. E. Carpenter \& V. H. Niem (Eds.), Guía FAO para la identificación de especies para los fines de la pesca, Pacífico centro-oriental. FAO, Roma, Italia.

Rivas, M. I. (1998). Ecología Trófica de algunas especies de los PNN Gorgona y Sanquianga (Tesis pregrado). Universidad del Valle, Cali, Colombia.

Rojas, P. A \& Zapata, L. A. (2006). Peces demersales del Parque Nacional Natural Gorgona y su área de influencia, Pacífico colombiano. Biota colombiana, 7(2): 211-244.

Ruiz-Ramírez, S., Lucano-Ramírez, G. \& Mariscal-Romero, J. (1997). Length-Weight relationships of softbottom demersal fishes from Jalisco and Colima states, Mexico. Naga, The ICLARM Quarterly, 20: 62-63.

UAESPNN. (1998). Plan de Manejo del Parque Nacional Natural Gorgona. Ministerio del Medio Ambiente, Bogotá, Colombia.

Udupa, K. S. (1986). Statistical methos of estimating the size at first maturity in fishes. Fishbyte, 4(2): 8-10.

Vazzoler, A. E. A. M. (1981). Manual de métodos para estudos biológicos de populações de peixes: Reprodução e crescimento. Programa Nacional de Zoologia, Brasília, Brasil.

Williams, T. (1974). "One-man" measuring board. In: FAO (E.), Manual of fisheries Science. Part 2 Methods of Resource Investigation and their application (pp. 63-68). FAO Fisheries Technical Paper No. 115, Rome, Italy.

Zapata L. A., Rodríguez, G., Beltrán, B., Gómez, G., Angulo, W., Gómez, A., Ramírez, M., Morales, Y., Hung, M., Herrera, J., Riascos, C. (1999). Prospección de los principales Bancos de Pesca en el Pacífico colombiano, durante noviembre de 1998. Bol. Científico INPA, 6: 111-175.

Zapata, L. A., Caicedo, J. A., Rojas, P., Gómez, S., Castellano-Galindo, G., Lora, A. M., Baos, R. \& Cuadros, L. (2010). Disminución del impacto por captura incidental de tortugas marinas con el uso de anzuelos curvos. Informe de País, Bogotá, Colombia.

Zorrilla, M. X. (1999). Aspectos reproductivos de algunos peces demersales del Parque Nacional Natural Gorgona, con énfasis en Gymnothorax sp. Aff. ecuatorialis (Pisces: Muraenidae) y Lutjanus argentiventris (Pisces: Lutjanidae) (Tesis de Grado). Universidad del Valle, Cali, Colombia. 
